\title{
Tiempo y singularización. Enfoques de las dinámicas relacionales del Dasein en elaboraciones de Heidegger entre 1927-1930
}

\author{
Luis Butierrez \\ Universidad Nacional de La Plata \\ luisbutierrez@yahoo.com.ar \\ https://orcid.org/0000-0001-9502-2234
}

Resumen: En el siguiente trabajo nos detendremos en un análisis y reconstrucción de las relaciones entre singularización y tiempo, respecto de sus especificaciones en torno al instante, a partir de una relación entre las elaboraciones de Heidegger en Sein und Zeit y su curso de 1929-1930. En ese marco, nos proponemos demostrar que sus consideraciones en torno a la relación dinámica Dasein-temporeidadsingularización profundizan la comprensión de fondo en la que se articulan, a partir de un cambio de acento que permite precisar con mayor claridad el concepto relacional de Dasein frente a las perspectivas subjetivistas tradicionales. De esta manera, buscamos desarrollar las relaciones entre singularización-tiempo en la obra de Heidegger y cotejar desde alli las condiciones para un marco interpretativo y recepción acorde al marco comprensivo de su obra.

Palabras clave: mismidad; instante; temporalidad; comprensión; acontecimiento

\begin{abstract}
Time and singularization. Approaches to the relational dynamics of Dasein in Heidegger's elaborations between 1927-1930". In the following work we will pause in an analysis and reconstruction of the relations between singularization and time, with respect to their specifications around the moment, from a relation between Heidegger's elaborations in Sein und Zeit and his course from 1929-1930. In this framework, we intend to show that his considerations around the dynamic relationship Dasein-temporality-singularization deepen background understanding in which they are articulated, based on a change of accent that allows the relational concept of Dasein to be specified more clearly, versus traditional subjectivist perspectives. In this way, we seek to develop the relationships between singularization-time in Heidegger's work and to compare the conditions for an interpretative and reception framework from there according to the comprehensive framework of his work.
\end{abstract}

Keywords: sameness; moment; temporality; understanding; event 


\section{Introducción}

En trabajos de Heidegger del período entre 1927 y 1930, podemos encontrar un conjunto de elaboraciones en torno al proceso de singularización, la situación y la temporalidad que plantean una transición respecto de los tratamientos subjetivistas tradicionales, especialmente en referencia a la tematización del rol y posición del existente humano en el marco de su situación y su época. Entre los debates inmediatos que estas cuestiones en la obra de Heidegger han generado, se destaca la interpretación de Sartre en El ser y la nada (1943), que bien puede entenderse como una tentativa por acentuar los aspectos prácticos de intervención directa en la historia (y en la constitución del sí mismo), sin ambigüedades ni embagues. Esta línea no solo se ha desarrollado entre otras recepciones de la época, sino también en distintos análisis y trabajos recientes respecto a estas cuestiones en la obra heideggeriana.

Tales lecturas e interpretaciones, en gran medida, han puesto el acento en el proceso de singularización en Sein und Zeit (SuZ), en especial, a partir de las relaciones entre instante (Augenblick), resolución (Entschlossenheit) y mismidad (Selbigkeit) ${ }^{1}$. En los últimos años podemos distinguir un interés creciente de articular estas cuestiones tanto con las elaboraciones de juventud de Heidegger como con sus trabajos en los textos de la Kehre ${ }^{2}$. En este recorrido, las divergencias en torno a la interpretación de la posición o estatuto práctico del Dasein en estas dinámicas aún mantienen vigente el debate. Incluso se actualiza la pregunta por el margen decisional del Dasein, en especial, a partir de análisis y lecturas en torno a las injerencias prácticas, las cuestiones éticas y las implicancias políticas en el marco del cambio de perspectiva hacia las dinámicas relacionales

\footnotetext{
1 Discutimos aquí con las lecturas de Falkenhayn von (Cf. Von Falkenhayn, K., Augenblick und Kairos. Zeitlichkeit im Frühwerk Martin Heideggers, Berlin: Duncker \& Humblot, 2003), Escudero (Escudero, J., "Ser y tiempo y el imperativo de una estética de la existencia: el Dasein como una obra de arte", en: Revista de Humanidades, 21 (2010a), pp. 13ss.) y Garrido (Garrido, Á., "Instante y situación. Problematización de la relación entre instante y situación en torno a Ser y tiempo de Martin Heidegger", en: Ágora. Papeles de filosofia, v. XXXVII, 2 (2018), pp. 57-63).

2 En este trabajo debatimos con los análisis respectivos de Escudero (Escudero, J., Heidegger y la genealogía de la pregunta por el ser, Barcelona: Herder, 2010) y Lythgoe (Lythgoe, E., "Disposición afectiva y temporalidad en Martin Heidegger entre 1927 y 1930", en: Revista de Filosofia Aurora, v. XXVI, 39 (2014), pp. 762-772).
} 
Dasein-ser en el horizonte del Ereignis ${ }^{3}$. En el marco de estas interpretaciones, se torna necesario circunscribir los aspectos que implican un desplazamiento de perspectiva entre estos momentos de la obra heideggeriana.

El siguiente artículo se inscribe en esta tradición de estudios del proceso de singularización en la obra de Heidegger, con la atención puesta en tales variaciones o desplazamientos. Específicamente, realizaremos un análisis y reconstrucción de las relaciones entre singularización y tiempo, en sus especificaciones en torno al instante, en el contexto de dos tramos fundamentales de la obra de Heidegger entre 1927-1930. Con ello nos proponemos demostrar que sus consideraciones en torno a la relación temporeidad-singularización desarrollan y profundizan su comprensión de fondo por medio de un cambio de acento que permite precisar con mayor claridad el concepto relacional de Dasein en las dinámicas de singularización y en sus diferencias respecto de las perspectivas subjetivistas tradicionales. En general, con estas lecturas buscamos cotejar la legitimidad y alcance de la pregunta por el estatuto práctico de la injerencia del Dasein respecto de sus relaciones con el sí mismo y la situación, en el marco de las elaboraciones y el discurso de Heidegger.

Para dar cuenta de ello, en primer lugar, nos detendremos en la reconstrucción y análisis de las relaciones entre el proceso de singularización y el instante en SuZ, destacando ciertas reelaboraciones en su curso de Marburgo de 1927. En este contexto, especificaremos los aspectos primarios y relacionales de tales dinámicas: la comprensión y relación con la muerte, el llamado de la conciencia y la articulación unitaria del tiempo en el instante, con el objeto de dar cuenta del carácter acontecimental que revisten tales tratamientos. En segundo lugar, analizaremos las relaciones entre singularización, instante y aburrimiento profundo en su curso de Friburgo de 1929-1930, para circunscribir continuidades y diferencias respecto de sus elaboraciones anteriores.

Por medio de este recorrido y análisis, nos proponemos precisar las relaciones entre singularización-tiempo en la obra de Heidegger y cotejar, desde allí, las condiciones para un marco interpretativo y recepción acorde al marco comprensivo de su obra. En vistas de ello, comencemos con sus principales elaboraciones de 1927.

3 De Lara, F., "El gesto político de las filosofias de Heidegger. Contribución a un debate actual", en: Veritas, 31 (2014), pp. 73-86; Calle Zapata, M., "Martin Heidegger y el intento por pensar la esencia de la técnica como una reorientación" en: Ethos, Revista Perseitas, v. IV, 1 (2016), pp. 60 ss.; y Di Pego, A., "En torno al pensamiento: la disputa de Hannah Arendt con Martin Heidegger, Tópicos”, en: Revista de Filosofía, 56 (2019), pp. 213-19; entre otros. 


\section{Relaciones entre singularidad y tiempo en la analitica de 1927}

Las elaboraciones en la época de SuZ se orientan hacia una descentralización de la voluntad y la racionalidad respecto de los abordajes tradicionales del existente humano y sus procesos de mismidad, aunque en tensión con la herencia comprensiva y discursiva de las perspectivas modernas del sujeto y la conciencia. Ello se pone especialmente de manifiesto en el análisis del pasaje de la impropiedad a la propiedad del Dasein, esto es, en el proceso de singularización (Vereinzelung) ${ }^{4}$ por el cual el existente adviene a sí mismo de un modo propio.

Si bien parte de las interpretaciones recientes reconstruyen estas cuestiones a partir de la consideración central y primaria del Dasein en estas dinámicas, las diversas estructuras co-intervinientes en las elaboraciones de finales de 1920 no excluyen la posibilidad de lecturas divergentes, especialmente si consideramos la constelación relacional que se articula en tales procesos. En el siguiente apartado nos proponemos desarrollar estas lecturas, por medio de un análisis de las elaboraciones respectivas en la analítica de Sein und Zeit (SuZ) y en ciertas articulaciones en su curso de Marburgo Die Grundprobleme der Phänomenologie (DGPh) de 1927, para contribuir con un desplazamiento de aquellas lecturas subjetivistas que han orientado parte de la recepción contemporánea de la obra heideggeriana.

Para dar cuenta de ello, primero nos enfocaremos en los marcos dinámicos y transicionales que se ponen de manifiesto en sus consideraciones sobre la comprensión propia, el precursar la muerte y el llamado de la conciencia en SuZ. Luego distinguiremos el concepto de instante con su específica comprensión de la temporeidad (Zeitlichkeit) originaria ${ }^{5}$, en vistas de sus continuidades y diferencias con especificaciones de su curso de Marburgo. Con ello nos proponemos cotejar en qué medida es posible distinguir aquí una dinámica acontecimental de la singularización del Dasein ${ }^{6}$, esto es, no reductible a sus aspectos decisionales o voluntarios, en vistas de un contraste con su Seminario de 1929-1930.

\footnotetext{
4 El verbo vereinzeln y el sustantivo Vereinzelung significan aislar y aislamiento (o separación). El uso que le da Heidegger en la época de SuZ remite a la vuelta del Dasein a sí mismo de un modo propio, en el marco de un proceso de pérdida y recuperación de sí en relación al mundo. Para dar cuenta de tal propiedad y diferencia, aquí lo traducimos por singularización.

5 Distinguimos aquí la temporeidad (Zeitlichkeit), como determinación del ser del Dasein por el tiempo y la temporalidad (Temporalität), en tanto determinación del ser mismo por el tiempo.

6 La categoría de acontecimiento se articula en diversos sentidos dentro de discursos y perspectivas contemporáneas. En este caso, el uso del término se halla inscripto en el campo semántico y comprensivo del Ereignis heideggeriano. Aquí destacamos su carácter irreductiblemente
} 


\subsection{Condiciones para la singularización}

En efecto, entre los parágrafos 29-32 de SuZ, Heidegger analiza un conjunto de estructuras relativas a la mismidad del Dasein a partir de su descripción del existenciario del ser-en (In-sein), lo cual permite poner de manifiesto su carácter irreductiblemente situado y arrojado en el mundo. Allí destaca que, al abrirse al mundo, el Dasein se abre a sí mismo y se comprende siendo. En este marco de simultaneidad Dasein-mundo, se ponen de manifiesto una multiplicidad de caracteres ontológicos constitutivos, entre ellos, la disposición afectiva (Befindlichkeit) y el comprender (Verstehen). Debido a este carácter entramado, ninguno de ellos puede dar cuenta aisladamente del todo de aperturidad (Erchlossenheit) del Dasein en su relación con el ser. En este sentido, podemos reconocer aqui una matriz relacional originariamente irreductible entre las estructuras del Dasein, en especial si consideramos las articulaciones entre sus dimensiones existencial-óntica y existenciaria-ontológica ${ }^{7}$, las cuales cierran la posibilidad de articular un enfoque subjetivista para estas elaboraciones ${ }^{8}$.

En el contexto de este entramado estructural, por un lado, la disposición afectiva como existencial refiere al estado de anímico (Stimmung) que pone al ser en su ahí $(D a)$, esto es, el ámbito situacional donde el Dasein ya está siempre dispuesto afectivamente de un modo u otro, incluso en su comprensión ${ }^{9}$. Por otro, con el comprender propio se pone de manifiesto existencialmente

relacional que permite contraponerlo al campo semántico de la metafísica de la subjetividad. En este sentido, sostiene un intérprete: "El Dasein no responde a qué es el ser humano, sino cómo se relaciona con ciertos fenómenos fundamentales que lo constituyen como tal" (Lythgoe, E., "Modos de ser y temporalidad en la analítica existenciaria", en: Revista de Filosofia, v. XXVII, 2 (2002), p. 263).

Heidegger, M., Sein und Zeit, Fráncfort: Klostermann,1927a, §203. Confróntese, además, con la interpretación de la bidimensionalidad (Zweidimensionalität) estructural del Dasein de Basso y Richardson: Basso, L., "Aportes para una interpretación de la estructura del Dasein de acuerdo a su carácter bidimensional" en: Areté. Revista de Filosofia, v. XXVI, 2 (2014), pp. 277ss; y Richardson, W., Heidegger: Through Phenomenology to Thought, La Haya: Martinus Nijhoff, 1967, pp. 49ss.

8 Cf. Vélez, G., "Peso de la existencia y dificultad hermenéutica en la génesis del pensamiento de Heidegger", en: Co-herencia, v. VI, 10 (2009), pp.120-123 y, del mismo autor, "La semilla del humanismo Moralidad y ontologia fundamental en Heidegger", en: Co-herencia, v. X, 20 (2014), pp. 127-136. Un enfoque crítico respectivo puede encontrarse en Burlando, G., "Un análisis interno del dasein ontológico de Heidegger: críticas externas de Edith Stein", en: Mirabilia: Revista Eletrônica de História Antiga e Medieval, 20 (2015), pp. 376ss.

9 En la década de 1920, Heidegger refiere a diversos aspectos en torno a la disposición afectiva: al estado de ánimo; el temple anímico (Gestimmheit) y el plural, estados de ánimo (Stimmungen). Como veremos, a partir de sus lecciones de la década de 1930 considera el temple de ánimo fundamental (Grundstimmung) en su carácter primario y anterior a toda dinámica de mismidad. Para un análisis comparativo, véase Santiesteban, L., "Heidegger: la relación de su pensar con la antropologia", en: Signos Filosóficos, v. VII, 13 (2005), pp. 82-84. 
el poder-ser (Sein-können) como modo de ser proyectivo: porque siempre se comprende, el Dasein como posibilidad arrojada puede extraviarse, malentenderse y recuperarse ${ }^{10}$.

Ahora bien, el Dasein ya siempre se ha proyectado y es tal proyectarse mientras existe, al mismo tiempo que se ha comprendido desde y hacia posibilidades articuladas a la propia situación. Precisamente desde este proyectar (Entwerfen), se comprende y se relaciona con los entes, los otros y con su sí mismo. En esta línea, Heidegger distingue la comprensión propia y auténtica, la cual requiere de ciertas condiciones, las cuales son analizadas en los dos primeros capítulos de la segunda sección de SuZ, en el marco de sus elaboraciones en torno a la muerte y la conciencia.

En primer lugar, destaca que mientras el Dasein es, se comporta en relación a su poder-ser-sí-mismo (Selbst-sein-können), develando con ello su permanente inconclusión hasta que no queda nada pendiente en su existencia. En este marco, Heidegger inscribe una vía para la asunción propia de la mismidad: con el ser-hasta-la-muerte (Sein zum Tode) del Dasein, se pone de manifiesto su posibilidad más propia.

Más en detalle: debido al carácter ineludible de inminencia, su muerte es la posibilidad de no-poder-existir-más que lo remite a su poder-ser más propio. Heidegger denomina precursar o adelantarse (Vorlaufen) a la muerte a este modo propio de venir a sí mismo y comprenderse. Sin embargo, aquí no se trata de una referencia velada a la voluntad: este inevitable estar arrojado hacia la muerte se le revela al Dasein con la angustia (Angst).

En línea con ello, el direccionamiento propio de este anticiparse o adelantarse no remite a una espera ni afán de ocuparse de la muerte, sino a comprenderla como posibilidad, interpretada y sobrellevada como tal, en el marco de un adelantarse hacia la posibilidad (Vorlaufen in die Möglichkeit) "de la inconmensurable imposibilidad de la existencia"11: se trata aquí solo de comprenderla como posibilidad, lo cual pone de manifiesto la muerte en su carácter radical.

Por esta vía, la muerte reivindica al Dasein como individual (als einzelnes), es decir, lo aísla hacia sí mismo ${ }^{12}$, permitiéndole comprender y elegir por primera vez en forma propia las posibilidades fácticas que están antepuestas a esta posibilidad insuperable. Con ello interrumpe la dependencia del Uno

${ }^{10}$ Heidegger, M., Sein und Zeit, §167.

${ }^{11}$ Heidegger, M., Sein und Zeit, §281.

12 Ibid., §283. 
(das Man), en tanto modo cotidiano de ser-con (Mit-sein) otros, al tiempo que lo vuelve sobre sí mismo en un modo propio. Este enfoque diferenciado de la tradición cartesiana por su carácter relacional y estructuralmente entramado se despliega de un modo más evidente con su abordaje en torno a las relaciones entre conciencia y singularización.

En efecto, nuestro autor también especifica que, para una modificación existentiva, primero el Dasein debe ser mostrado a sí mismo en cuanto posibilidad. Ello puede lograrlo por medio de la voz de la conciencia (Stimme des Gewissens), fenómeno que Heidegger aborda a nivel ontológico entre los parágrafos 54 a 60 de SuZ destacando la dimensión de alteridad inherente a las dinámicas de singularización ${ }^{13}$.

En estas elaboraciones la conciencia es una instancia en que se comunica la posibilidad de un poder-ser propio, en el marco de una dinámica de llamado y respuesta, no como resultado de una decisión ni expresión de la voluntad, sino en el marco de una dinámica relacional: la conciencia abre, da a entender algo y se revela como una llamada (Ruf), es decir, como apelación al Dasein a hacerse cargo del propio poder-ser sí mismo. A esta llamada corresponde luego el escuchar y la comprensión de un querer-tener-conciencia, esto es, un pliegue volitivo secundario que repercute en la resolución como acto existentivo, al modo de una intimidación a despertar que Heidegger distingue como el más propio ser-culpable. Esta noción remite aquí a un ámbito originario: en su existencia arrojada, el Dasein no se ha puesto a sí mismo. En ese sentido, no le está dado ser el soberano de su existencia ni crear sus posibilidades ex nihilo, sino que está entregado a ellas en su facticidad, es decir, en/desde la situación y relaciones respectivas.

Por ello, Heidegger distingue que se trata aquí de una interpretación respectiva de sí mismo que se aplica, sin embargo, sobre sí mismo, es decir, viene de mi propia conciencia como un más allá de mí que llama a la propia mismidad ${ }^{14}$. Esta duplicación o pliegue ha suscitado lecturas que distinguen aquí un sesgo de extranjeridad inherente a estas dinámicas a la conciencia, que aquí entendemos como una dimensión de alteridad en las dinámicas de singularización.

\footnotetext{
${ }^{13}$ Respecto de la comprensión de la alteridad en estas elaboraciones, cf. Butierrez, L. F., "Caminos hacia la alteridad. La comprensión del otro en las elaboraciones de Heidegger en torno a Sein und Zeit", en: Revista Anales del Seminario de Historia de la Filosofia, v. XXXVII, 1 (2020), pp. 105ss.

${ }^{14}$ Heidegger, M., Sein und Zeit, §294.
} 
En suma, por medio de estas condiciones es posible una comprensión propia respecto a sí, logrando convertirse en el fundamento arrojado de su propia finitud. Desde aquí, se abre una modalización que atraviesa el complejo dinámico y simultáneo de las estructuras cooriginarias del Dasein respecto a las relaciones con los entes, con los otros Dasein, con las significaciones y con el lenguaje, las cuales ponen en tensión las estructuras de su cotidianidad y descubren una dimensión contingente, dinámica, no clausurable ni plenificable. Ello se articula con la distinción de Ferrer Santos ${ }^{15}$ (2001) en lo que respecta a la posible autodeterminación del Dasein: en SuZ, el margen decisional remite a un término situacional primario que abre y determina toda decisión posible ${ }^{16}$. En tal sentido, tal marco decisional del Dasein se articula en una conducta derivada y relacional, tal y como se halla clarificado en sus elaboraciones respectivas sobre la temporeidad.

\subsection{La temporeidad del instante: ¿decisión o acontecimiento?}

En el contexto de estas dinámicas, la interpretación radical de los éxtasis temporales se vincula con aquel estado de resuelto: en el venir hacia sí-mismo desde la posibilidad propia surge lo que Heidegger distingue como el instante (Augenblick). Esta modalidad temporal de presente propio devela la situación donde el estar-resuelto se efectiviza.

Específicamente, las dinámicas de la temporeidad originaria que se dan en el instante remiten a una relación articulada entre los tres éxtasis temporales, tal y como Heidegger las analiza entre los parágrafos 65 a 68 de SuZ. En este marco, especifica que la dimensión extática de futuro del Dasein se abre cuando se resuelve al modo de un advenir propiamente sí mismo desde su poder-ser y su anticiparse a la muerte, lo cual revela el fenómeno originario del porvenir (Zukunft), no reductible a un mero futuro tal y como es comprendido en la cotidianidad.

Este carácter de venidero (Zukünftig) co-implica hacerse cargo de la condición de arrojado (Geworfenheit) y hacerlo desde lo que hemos sido. El haber-sido (Gewesenheit) refiere también al ámbito desde el cual se nos abre el presente en el que nos relacionamos con los entres y los otros Dasein. Así, todo anticiparse lleva en sí un pasado propio que Heidegger llama re-iteración

\footnotetext{
${ }^{15}$ Cf. Ferrer Santos, U., "De la autodeterminación del existente a la aceptación del ser en la Analítica existencial heideggeriana", en: Investigaciones fenomenológicas, 3 (2001), pp. 179-193.

${ }^{16}$ Cf. ibid., pp. 179-193.
} 
o repetición (Wiederholung) ${ }^{17}$ : la autenticidad requiere que el Dasein asuma el fárrago del ente que ya es, proyectando las posibilidades fácticamente posibles (en relación a lo sido del Dasein), para lanzarse hacia el futuro y encaminarse hacia la muerte.

De este modo, los tres éxtasis se inter-penetran conjuntamente y el modo en que lo hacen permite dar cuenta de la posibilidad de la existencia propia e impropia ${ }^{18}$. En este sentido, el instante de la resolución del Dasein hacia la singularización es el presente como anticipar-reiterante, que temporaliza el conjunto de la existencia, abriendo la situación en una modalidad integral de los éxtasis, hacia la muerte como posibilidad. Así, desde la perspectiva del Dasein el anticiparse a la muerte es la condición temporal última y originaria tanto del fenómeno del instante como de la apertura de la situación, lo cual permite no identificar la resolución con la decisión (Entschluss) del Dasein. Con ello se pone de manifiesto lo que puede caracterizarse como el fenómeno temporal intrinseco del instante en SuZ ${ }^{19}$.

Ahora bien, el instante no necesariamente tiene que manifestarse como presente puntual en la modalidad del ahora. En efecto, en el presente propio, el Dasein echa una mirada (Blick) al mundo en el que él es y el modo en que lo $e^{20}$, lo cual requiere un tiempo de comprender y una demora ante la situación o las posibilidades, diferenciándose así de aquello que implica el ahora en su carácter repentino y puntual ${ }^{21}$. En tal sentido, ¿es posible plantear aquí un margen decisional o racional implícito para estas relaciones entre instante y singularización?

Con el instante se revela una apertura ontológicamente anterior a todo comportamiento teórico y práctico, donde toda resolución y proyección solo es asumible en lo previamente abierto. Por ello, Heidegger busca evitar el uso del término actuar (Handeln): la resolución no queda ligada a un acto de voluntad, sino que se caracteriza por una dinámica relacional en el marco de la posibilidad

\footnotetext{
${ }^{17}$ Heidegger, M., Sein und Zeit, §355.

${ }^{18}$ Ibid., §345.

19 Aquí seguimos el análisis y la interpretación del instante realizadas en: Ward, K., Augenblick. The Concept of the 'Decisive Moment' in 19th - and 20th Century Western Philosophy, Aldershot/ Burlington: Ashgate Publishing, 2008, pp. 117ss.

${ }^{20}$ La etimologia del término alemán Augenblick se acerca a la significación de mirada, parpadeo o golpe de vista. Al respecto, véase la segunda parte de la Introducción en: Von Falkenhayn, K., Augenblick und Kairos. Zeitlichkeit im Frühwerk Martin Heideggers. Cf. Heidegger, M., Die Grundprobleme der Phänomenologie, Fráncfort: Klostermann,1927b, §344.

${ }^{21} C f$. Garrido, Á., "Instante y situación. Problematización de la relación entre instante y situación en torno a Ser y tiempo de Martin Heidegger", en: Ágora. Papeles de filosofia, v. XXXVII, 2 (2018), p. 61.
} 
proyectiva ${ }^{22}$. En tal sentido, el instante no puede ser producido, sino solo esperado y asumido. Para el Dasein, la situación como marco de propiedad tiene que hacérsele presente para poder asumirla y resolverse al modo de una respuesta.

Este marco relacional irreductible de las dinámicas de singularización se halla puntualizado en el curso de Marburgo de 1927 Die Grundprobleme der Phänomenologie (DGPh). Allí podemos encontrar un tratamiento de estas relaciones entre mismidad e instante a partir de sus distinciones en torno a la temporeidad desde los parágrafos 19 y 20 de la segunda parte del Curso, donde nuestro autor da un paso de mayor clarificación al respecto, en especial, al momento de desarrollar sus consideraciones del carácter horizontal de la temporalidad, solo esbozado en $\mathrm{SuZ}$ en su articulación de la temporeidad del Dasein $^{23}$.

En el contexto de las tematizaciones de la relación irreductible entre el fenómeno del tiempo, el concepto de mundo y la estructura del Dasein ${ }^{24}$, nuestro autor parte de un fenómeno temporal para dar cuenta de la dinámica de mismidad: la espera (erwarten $)^{25}$. Desde la comprensión impropia de sí vinculada a ello, Heidegger contrapone la dinámica temporal de singularización, en especial, a partir de un análisis del horizonte de los éxtasis temporales, al destacar que cada uno de ellos está abierto de un modo determinado. Respecto de esta apertura, subraya que mediante el poder-ser el Dasein es arrebatado (entrückt) fuera de sí hacia cada modalidad propia de los éxtasis. En tal sentido, especifica que "el arrebatamiento abre y mantiene abierto ese horizonte (Die Entrückung öffnet und hält diesen Horizont offen)"26.

Con esta distinción vinculada a una disposición afectiva, la temporeidad es entendida como extático-horizontal (ekstatisch-horizontal) en línea comprensiva con las dinámicas especificadas en $\mathrm{SuZ}$, pero poniendo mayor énfasis en el aspecto relacional, esto es, en un enfoque más allá de la perspectiva del Dasein. Es precisamente por la vía de este desplazamiento respecto del

${ }^{22}$ En sus cursos de finales de la década de 1920 pasa del uso de resolución en SuZ al de decisión (Entscheidung), buscando con ello contrarrestar las resonancias subjetivistas. Al respecto, véase Santiesteban, L., Heidegger y la ética, Ciudad de México: Aldus, 2009, p. 95.

${ }^{23}$ En SuZ, la temporeidad incluye el horizonte temporal de ser de todo ente en general, conformando la esencia del tiempo originario. Al respecto, véase Von Herrmann, F-W., La segunda mitad de Ser y Tiempo. Sobre los problemas fundamentales de la Fenomenología de Heidegger, Madrid: Trotta, 1997, pp. 39ss.

${ }^{24}$ Heidegger, M., Die Grundprobleme der Phänomenologie, §307.

${ }^{25}$ Aquí el Dasein proyecta su poder-ser a partir de las cosas y desde allí se comprende, quedando de este modo determinado por el éxito o fracaso, factibilidad o infactibilidad del trato con las cosas. Cf. ibid., §§319ss.

${ }^{26}$ Ibid., p. 378 [§322]. 
enfoque de la analítica de $\mathrm{SuZ}$ por la cual Heidegger profundiza y desarrolla su comprensión relacional y acontecimental de la singularización en su curso de Friburgo de 1929.

\section{Singularidad e instante en 1929-1930}

En el curso de Friburgo Die Grundbegriffe der Metaphysik Welt Endlichkeit - Einsamkeit (1929-30) (GbM), Heidegger despliega un análisis en torno a la relación entre la mismidad del Dasein y la temporeidad que presenta diferencias y continuidades respecto de su perspectiva articulada en SuZ, especialmente respecto de las relaciones en las dinámicas de singularización que hemos analizado.

En dicho seminario, podemos situar lo que en los estudios ya clásicos de Kawahara ${ }^{27}$ y Van Dikj ${ }^{28}$ han comprendido como la experiencia epocal de la indiferencia del ser. Desde ese enfoque, distinguimos dos conceptos principales desde los cuales Heidegger despliega estas cuestiones: la finitud y la singularización del Dasein en relación al "retiro a la soledad en la que todo hombre llega por vez primera a la proximidad de lo esencial de todas las cosas, al mundo" 29 , donde cada uno está por sí mismo ante el conjunto de los entes. Desde aquí, el concepto de mundo orienta los desarrollos de este seminario, en especial, desde la perspectiva del Stimmung fundamental del aburrimiento profundo, un temple que permite poner de manifiesto el mundo en su totalidad y al sí mismo propio.

En los primeros parágrafos, Heidegger plantea con claridad el cambio de acento que presenta en estos análisis, al tomar un temple anímico fundamental como marco desde el cual plantear una experiencia y su recorrido filosófico. Para ello se propone retomar y preservar el pasado significativo de la tradición griega, anterior a la formación de las Escuelas, donde el inquirir filosófico y su articulación conceptual co-implican un arrebatamiento originario por parte de aquello mismo que se interroga. En sus antipodas se halla el ideal de conocimiento matemático correlativo al concepto de verdad de cuño platónico, poco vinculante para el hombre respecto a su existencia dinámica y cambiante ${ }^{30}$.

${ }^{27}$ Cf. Kawahara, E., "Heideggers Auslegung der Langweile", en: Wisser, R. (ed.), Unterwegs im Denken, Friburgo/Múnich: Karl Alber, 1987, pp. 90ss.

${ }^{28}$ Cf. Van Dijk, R., "Grundbegriffe der Metaphysik. Zur formalanzeigenden Struktur der philosophischen Begriffen bei Heidegger", en: Heidegger Studien, 7 (1991), pp. 92-110.

${ }^{29}$ Heidegger, M., Die Grundprobleme der Phänomenologie, §29.

30 Ibid., $\S \S 41$ ss. 
Precisamente este marco experiencial caracteriza aquellas dos líneas de desarrollo que atraviesan la mayor parte del curso.

A diferencia de ciertas lecturas que ven en este curso un desplazamiento temático que pasa de centrarse en la figura del sí mismo como individual, al temple colectivo de una época ${ }^{31}$, en este apartado argumentaremos que aquí se da una profundización de las relaciones entre mismidad propia y temporeidad con un énfasis más claro en el aspecto relacional de la dinámica y no a partir del despliegue de uno de los elementos o estructuras que la componen. En este sentido, sea la figura temática del sí mismo o la del temple colectivo de una época, se trata en todo caso del ser temporal del Dasein, cuya relación específica con la temporeidad es más originaria que toda determinación subjetiva, el yo o la conciencia.

A continuación, nos detendremos en el tercer modo o forma que Heidegger distingue de aquel temple para circunscribir las relaciones entre temporeidad y singularizaciones alli inscriptas. Por esta vía, nos proponemos cotejar las continuidades y diferencias respecto a las elaboraciones anteriores, en vistas del cambio de enfoque hacia la relación irreductible Dasein-Ser que caracteriza sus textos del período de la Kehre.

\subsection{Las formas del aburrimiento y sus relaciones con la temporeidad}

Desde el parágrafo 16 del curso, nuestro autor despliega sus análisis subrayando que el aburrimiento profundo (tiefe Langeweile) es un temple de ánimo fundamental, cuya modalidad vincular cotidiana se da en el modo de la huida, esto es, correlativa con una intervención práctica que se propone detenerlo, eliminarlo o hacer que se duerma y, con ello, mantenerlo a su alcance bajo cálculo. Frente a esta tendencia de adormecimiento, nuestro autor se aboca a la tentativa de despertar el temple de ánimo, es decir, hacer que esté despierto y preservarlo del dormirse (Einschlafen). Con esta propuesta práctica, nuestro autor enfatiza el aspecto no voluntario de esta dinámica relacional: el temple condiciona la experiencia, al tiempo que lo dado en ella modela el temple.

En este contexto, se pone de manifiesto una experiencia pura del tiempo. La dimensión temporal implicada allí se torna evidente en la expresión alemana que literalmente significa: el momento (Weile) que se hace largo (lang), lo cual ya prefigura una diferencia fundamental con el instante (Augenblick), cuyo

\footnotetext{
${ }^{31}$ Esta tesis puede encontrarse en Catoggio, L., y D. Parente, "Angustia y aburrimiento. Reflexiones sobre el desplazamiento temático en el primer Heidegger”, en: Ergo, 22-23 (2008), pp. 14-21.
} 
término sugiere una temporalidad atravesada por las dinámicas temporales de la finitud humana.

Específicamente, el procedimiento de Heidegger tiene como hilo conductor indagar en el ámbito donde nos oponemos al temple: el pasatiempo (Zeitvertreib). De este modo, busca mantenerse en la inmediatez de la experiencia cotidiana sin imponer acciones directas, sino solo determinar el temple y, luego, despertarlo.

Por esta vía, distingue tres formas de aburrimiento desde expresiones/ experiencias correlativas: el aburrirse con algo (das Gelangweiltwerden von etwas); el aburrirse en/por algo (das Sichlangweilen bei etwas) y el uno se aburre (o me aburro) (es ist einem langweilig), en referencia a un aburrirse sin más (das Sichlangweiligen). Tales distinciones permiten dar cuenta de los diversos grados de profundidad en la que implican a la existencia. En el primer caso, parte del ejemplo cotidiano de llegar antes de horario a la estación de tren; en el segundo, de la asistencia a una velada para distraerse del trabajo, y en el tercero, destacando la dificultad que implica su determinación, esboza el caso de caminar por una gran ciudad un domingo por la tarde. Desde cada uno de ellos, indaga en aquel pasatiempo para oponerse al aburrimiento. Los dos momentos estructurales de estas tres formas de aburrimiento son el dar largas (Hingehaltenheit) del tiempo (como oprimir de este) y el ser dejados vacíos (Leergelassenheit) por las cosas (o por el ente en su conjunto). Por medio de ambos, Heidegger pone en evidencia las relaciones del temple fundamental con el mundo y el sí mismo.

Como vimos, el carácter coercitivo y sustractivo del Dasein en su cotidianidad por parte de un temple ya se encuentra prefigurado en sus elaboraciones anteriores en torno a la angustia, como una de las condiciones para los procesos de singularización ${ }^{32}$. No obstante, la especificidad del aburrimiento reside en su modo de proporcionar herramientas para saber moverse en la profundidad de la existencia del Dasein ${ }^{33}$, por lo cual Heidegger destaca aspectos prácticos ligados a este temple fundamental: un saber-hacer que orienta el procedimiento del despertar este temple de ánimo fundamental. En todo caso, la esencia

\footnotetext{
${ }^{32}$ Además de sus elaboraciones en el parágrafo 40 de SuZ, véase su conferencia Was ist Metaphysik? de julio del mismo año. Estos trabajos forman parte de una amplia literatura de análisis comparativo entre la angustia y el aburrimiento como temples que revelan el mundo y forman parte de las dinámicas propias de retorno a sí mismo. Al respecto, véanse los análisis de Pocai, Basso y Escudero: Pocai, R., Heideggers Theorie der Befindlichkeit, Friburgo/Múnich: Karl Alber Verlag, 1996, pp. 193ss.; Basso, L., "Aportes para una interpretación de la estructura del Dasein de acuerdo a su carácter bidimensional", pp. 287ss; y Escudero, J., "En torno al aburrimiento (o de la importancia de los estados de ánimo en la filosofia de Heidegger)", en: Revista Estudio, 18 (2014), pp. 36-40.

${ }^{33}$ Cf. Heidegger, M., Die Grundprobleme der Phänomenologie, §171.
} 
del aburrimiento le permite indagar en la esencia oculta del tiempo y sus conexiones mutuas. Esta imbricación entre aburrimiento-temporeidad-Dasein se profundiza a partir del tercer modo de aburrimiento, alli donde se expone su relación con el sí mismo, aunque al modo de una no-relación.

\subsection{El instante en el "Uno se aburre"}

En efecto, con la tercera modalidad de aburrimiento se expresa un ámbito de lo impersonal donde se suspende la mismidad propia; al tiempo, se abre el horizonte temporal de su existencia en el mundo. Aquí, la disolución de las relaciones con el ente se sigue de la disolución del sentido o el ser del conjunto del ente, lo cual permite experimentar la existencia como puro tiempo. En este contexto, nuestro autor circunscribe la emergencia de cierta interpelación que fuerza al Dasein a la escucha por medio de cierta coerción que reconoce como el rasgo distintivo de todo lo auténtico de la existencia y la libertad ${ }^{34}$. Con ello, Heidegger subraya el carácter relacional de la decisión del Dasein, en clara continuidad con sus especificaciones de la conciencia en SuZ.

Más en detalle: con el aburrimiento profundo, la indiferencia atraviesa tanto las cosas como el sí mismo. El Dasein se ve en cierta medida depuesto de la personalidad cotidiana y detenido en su conjunto, dándose una equivalencia con lo ente. Con ello, el sí mismo de la existencia se encuentra empobrecido en su singularidad. Es precisamente esta radicalización del abandono y la dejadez lo que pone de manifiesto un anunciamiento.

Así, el aburrimiento profundo revela el vértice único de lo posibilitador en general. Con él se manifiesta la vacuidad (Leergelassenheit) de puro horizonte temporal en el cual se encuentra lo ente en su conjunto. Junto a ello, los éxtasis temporales pierden su unidad en una desarticulación que los diluye en un mero horizonte de tiempo: una temporalidad que se muestra aquí como horizonte vacío donde puede acontecer todo suceso. En el marco de esta radicalización, Heidegger distingue el anuncio de lo propiamente posibilitante: se abre como horizonte de toda posibilidad y, con ello, revela el ser-tiempo del Dasein.

Ahora bien, ¿qué lugar ocupa el instante y la resolución del Dasein en el marco de esta manifestación horizontal de la temporalidad? Al igual que en sus elaboraciones de 1927, nuestro autor destaca que el Dasein se resuelve en el instante, allí donde la situación se abre. No obstante, el impulso para esta dinámica ya no es distinguida en la articulación de los éxtasis, tal como especifica en este fragmento: “...este resolverse (Sichentschließen) del Dasein en

34 Cf. ibid., §178. 
su mismidad, es decir, a ser en cada caso en medio de lo ente lo determinado que le es encomendado a ser, este resolverse es el instante. ¿Por qué? El Dasein, al fin y al cabo, no es algo presente entre otras cosas, sino que la manifestabilidad (Offenbarkeit) de todo el horizonte temporal la coloca en medio de lo ente" 35 .

Aquí la resolución del Dasein se ve conminada desde el horizonte temporal, donde el Dasein puede hacer uso del tiempo en tanto instante que propiamente la posibilita. Precisamente es alli donde se permite definirlo: "El instante no es otra cosa que la mirada de un estar resuelto en el que toda la situación de un actuar se abre y se mantiene abierta"36. De esta manera, tanto el instante, como el Dasein y su acción respectiva se hallan perdidos y detenidos por el aburrimiento. Anulado el Dasein en su capacidad de sustraerse al horizonte temporal (que se manifiesta cuando se le deniega lo ente en su conjunto), se ve expulsado (gebannt) a la amplitud de ese horizonte y, con ello, forzado al instante como vértice de lo propiamente posibilitador (die Spitze des eigentlich Ermöglichenden), en el mismo punto en que se pierde el vértice del instante.

Así, al mantenerse suspendido (hingehalten) en el horizonte temporal como unidad indiferenciada, el tiempo se revela como lo posibilitante y el Dasein se ve impelido a quebrar ese tiempo indiferenciado del que está cautivo, con lo cual se resuelve y singulariza. Aquí, la temporeidad del instante quiebra este vacío del tiempo, abre la situación y lleva al Dasein a determinarse a sí mismo en relación con ella ${ }^{37}$. Sin embargo, lo hace en segunda instancia: es precisamente la opresión del aburrimiento profundo en nuestra existencia y la anulación del horizonte temporal lo que indica y abre el camino al sí mismo propio.

\subsection{Algunos enfoques de estas dinámicas de singularización}

De esta manera, podemos reconocer aquí cierta continuidad y diferencia respecto de la modalidad de acceso a la mismidad propia en estos seminarios de 1929-1930. En este curso de Friburgo, la negación de todo ente en su conjunto manifiesta con el aburrimiento, proviene y se dinamiza desde horizonte temporal. No obstante, aquí entendemos que este cambio de acento en la relación entre situación e instante se articula en una perspectiva orientada a la relación Dasein-Ser. En todo caso, no deja de inscribirse en una relación desde la cual se articulan sus dinámicas: el aburrimiento y el horizonte temporal son los ámbitos primarios para dar cuenta de las mismas.

35 Ibid., §192. El énfasis es nuestro.

${ }^{36}$ Ibid., §193. Además, cf. Heidegger, M., Sein und Zeit, §65.

37 Cf. Heidegger, M., Die Grundprobleme der Phänomenologie, §194.

ARETÉ Revista de Filosofía, v. XXXIII, 1, 2021 / e-ISSN 2223-3741 
Frente a ello, podemos encontrar lecturas que interpretan la singularización a la que conduce este análisis que no incide en el propio concepto de temporeidad, como sí sucede en SuZ. En tal sentido, el instante pasaría de cumplir un papel metodológico a uno de corte ético-político, pues en este seminario el horizonte no sería el único componente del tiempo ${ }^{38}$. Esta interpretación parece enfocada a partir de un deslinde de la articulación relacional Dasein-ser. Frente a ello, las consideraciones del instante y la resolución en este trabajo de Heidegger continúan poniendo al Dasein en un rol derivado en el marco de estas dinámicas. Es precisamente esta continuidad con SuZ lo que permite desarrollar lecturas que reconocen aquí un margen decisional para el Dasein en los procesos de singularización, al modo de una respuesta ante aquello que el aburrimiento profundo le revela ${ }^{39}$. No obstante, ello también puede leerse como una dependencia de las modificaciones temporales, lo cual produciria un borramiento de un auténtico margen decisional del Dasein. En tal sentido, Garrido interpreta que es la situación la que determina o condiciona la receptividad del instante, tanto en $\mathrm{SuZ}$ como en el seminario de 1929-193040.

A nuestro entender, estas lecturas e indagaciones continúan de algún modo articuladas en el campo comprensivo de la metafísica de la subjetividad. A diferencia de ello, una lectura de estas dinámicas enfocada desde el concepto de Dasein permite reconocer un entramado relacional entre estructuras y existenciarios que dificilmente podría reducirse a una perspectiva parcial o fragmentada. Si el Dasein es esa relación temporal, toda dinámica co-implica su injerencia de algún modo. Precisamente por ello, puede sostenerse que estas elaboraciones de 1929-1930 parecen desestimar la vertiente racional-reflexiva del marco decisional del Dasein ${ }^{41}$, dejando en evidencia el carácter originario e irreductible de la relación Dasein-Ser ${ }^{42}$.

${ }^{38}$ Cf. Lythgoe, E., "Disposición afectiva y temporalidad en Martin Heidegger entre 1927 y 1930", pp. 771 ss.

${ }^{39}$ Cf. Redondo, P., Filosofar desde el temple de ánimo: la 'experiencia fundamental' y la teoría del 'encontrarse' en Heidegger, Salamanca: Ediciones Universidad de Salamanca, 2005, p. 191.

${ }^{40}$ Cf. Garrido, Á., "Instante y situación. Problematización de la relación entre instante y situación en torno a Ser y tiempo de Martin Heidegger", pp. 68ss.

41 Aun así, Heidegger también distingue en este curso el modo en que el hombre se apropia reflexivamente de sí mismo, respecto del animal que no supone una personalidad, ni reflexión ni conciencia, sino solo propiedad (Eigentum) en su relación de mismidad. Con ello, distingue la peculiaridad (Eigentümlichkeit) como el carácter propio de la persona en su modo de poseerse a sí misma (cf. Heidegger, M., Die Grundbegriffe der Metaphysik. Welt - Endlichkeit - Einsamkeit, Fráncfort: Klostermann, 1929-1930, §285.

42 En este sentido, un intérprete sostiene: "Esa donación no implica deuda y, sin embargo, compromete a quien la recibe en forma de un acontecimiento que se apropia de nosotros. Este es el sentido que cabe dar a la expresión Ereignis en cuanto fenómeno que señala la originaria 
Con este enfoque, podemos reconocer alli una figura de la tentativa heideggeriana de contraponerse a la voluntad de dominio y de cálculo, como propia de la tradición subjetivista, en el marco de su comprensión de las relaciones prácticas y dinámicas con el sí mismo. Una orientación que desde la década de 1930 articula con mayores especificaciones relativas al mundo de la técnica y sus consecuencias de época.

\section{A modo de conclusión}

A continuación, realizaremos algunas puntualizaciones de este recorrido por las relaciones entre singularización y temporeidad en elaboraciones de Heidegger entre 1927 y 1930, con el objeto de circunscribir con precisión nuestras lecturas de partida, en el marco de la pregunta respecto de la posición o rol del Dasein en las dinámicas respectivas. Con ello buscaremos dar cuenta de ciertas condiciones que entendemos necesarias para un abordaje apropiado de estas cuestiones en la obra de Heidegger.

En primer lugar, distinguimos lo que denominamos como la perspectiva acontecimental y los aspectos de alteridad en el proceso de singularización en $\mathrm{SuZ}$, por medio de las consideraciones de Heidegger en torno al adelantarse a la muerte, la comprensión propia y el llamado de la conciencia. En tal contexto, reconstruimos las relaciones temporales en el instante, desde el cual el Dasein realiza el pasaje de la impropiedad a la propiedad. Aquí, vimos que el marco temporal se ve unificado y articulado: desde allí se abre la situación y se dinamiza el pasaje de la mismidad en la cotidianidad.

A nuestro entender, esta cuestión requiere una perspectiva acorde con la conceptualidad de la obra de Heidegger: un enfoque desde la categoría de Dasein permite comprender el carácter irreductiblemente relacional de este proceso, aun cuando Heidegger lo disgregue y especifique en sus partes componentes en el marco de la analítica. Por ello, subrayamos que la dinámica de singularización se articula a partiry desde la relación Dasein-instante-situación. Precisamente, en este sentido, distinguimos una dinámica del orden del acontecimiento y no de la sustancia en el marco de la imbricación entre instancias que, al articularse,

copertenencia, la fusión indisoluble de ser y hombre. El ser es quien posibilita el aparecer de las cosas y de los hombres, pero simultáneamente el ser como iluminación no se produciría sin el hombre" (Escudero, J., "En torno al aburrimiento (o de la importancia de los estados de ánimo en la filosofia de Heidegger)", p. 42). 
se reconfiguran mutuamente ${ }^{43}$. Aun asi, el carácter temporal del instante en $\mathrm{SuZ}$ permite pensar un margen decisional o racional que interviene en el despliegue de tal articulación.

En segundo lugar, vimos el modo en que Heidegger desplaza el carácter liminar de ese enfoque en su curso de 1929-1930, hacia una perspectiva heterónoma desplegada a partir de un ámbito más originario que toda articulación subjetiva, desde el yo o la conciencia. Ello implica una profundización o radicalización de la comprensión de $\mathrm{SuZ}$ a partir del cambio de enfoque que le permiten el análisis del aburrimiento profundo y el desplazamiento en la consideración del horizonte temporal en las dinámicas del instante. Con ello, el carácter acontecimental-relacional con el que Heidegger reviste estas elaboraciones se torna más evidente. En especial, vimos que en la tercera forma del aburrimiento la resolución del Dasein es derivada desde el horizonte temporal, el cual se manifiesta vacío y extendido de un modo paradójico: en el mismo momento en que el Dasein y el discurrir temporal se ven anulados, se revela el instante como lo posibilitador. Con ello se pone de manifiesto la ligazón irreductible Dasein-tiempo que abre la totalidad del mundo.

En esta forma de abordar la singularización, Heidegger subraya su carácter experiencial sin un sustrato sustancial como fundamento y motor de la dinámica. De esta manera, el curso marca un tránsito en los análisis de Heidegger hacia una perspectiva relacional, al modo de una tentativa de abandono del enfoque desde el Dasein en la década de 1920 y sus vinculaciones con la metafísica de la subjetividad.

Por ello, sostenemos que en estas elaboraciones consideradas entre 19271930 puede distinguirse una continuidad y desarrollo de la comprensión de las dinámicas de singularización y las consideraciones en torno al rol o posición del Dasein, en especial a partir de un cambio de acento que clarifica y evidencia el marco relacional desde el cual Heidegger piensa y analiza estas dinámicas. En tal sentido, entendemos que una parte de la recepción de estas cuestiones en la obra heideggeriana ha planteado un enfoque desde el marco semántico

\footnotetext{
43 En este sentido, nos diferenciamos de las lecturas que remiten este carácter acontecimental a los trabajos posteriores a 1930. Como en esta lectura: "lo que le da a las cosas su verdadera y última fisonomía no sería para este pensador algo perteneciente al plano social y político, sino algo previo: radicalmente individual en el primer Heidegger; impersonal y no humanamente determinable en el segundo. En el Heidegger posterior a la Kehre el papel de esa instancia configuradora lo juega el Ser en su acontecimiento, al que los humanos no responden individualmente sino en cuanto pueblos" (De Lara, F., "El gesto politico de las filosofias de Heidegger. Contribución a un debate actual", p. 82).
} 
del concepto de sujeto o de individuo, esto es, desplegando interpretaciones deficitarias generalmente ligadas a una excesiva disociación de momentos o estructuras respectivas.

En tal sentido, vemos factible problematizar la legitimidad de la pregunta por el estatuto práctico del Dasein en el contexto de estos pasajes de su obra, especialmente si consideramos que el propio discurso de Heidegger, su conceptualización y su modalidad relacional dinámica con el lenguaje han establecido ciertas coordenadas o condiciones para un abordaje pertinente de su obra. Entre ellas, quedan excluidas las perspectivas subjetivistas tradicionales y su lenguaje respectivo.

Recibido: $12 / 08 / 2020$

Aceptado: 18/03/2021

\section{Bibliografia}

Escudero, J., "Ser y tiempo y el imperativo de una estética de la existencia: el Dasein como una obra de arte", en: Revista de Humanidades, 21, (2010a), pp. 9-29.

Escudero, J., Heidegger y la genealogía de la pregunta por el ser, Barcelona: Herder, 2010.

Escudero, J., "En torno al aburrimiento (o de la importancia de los estados de ánimo en la filosofia de Heidegger)", en: Revista Estudio, 18 (2014), pp. 33-43.

Agamben, G., Lo abierto, el hombre y el animal, Costa, F. y E. Castro (trads.), Buenos Aires: Adriana Hidalgo Editora, 2016.

Basso Monteverde, L., "Aportes para una interpretación de la estructura del Dasein de acuerdo a su carácter bidimensional" en: Areté. Revista de Filosofía, v. XXVI, 2 (2014), pp. 273-293.

Burlando, G., "Un análisis interno del dasein ontológico de Heidegger: críticas externas de Edith Stein", en: Mirabilia: Revista Eletrônica de História Antiga e Medieval, 20 (2015), pp. 365-82.

Butierrez, L.F., "Caminos hacia la alteridad. La comprensión del otro en las elaboraciones de Heidegger en torno a Sein und Zeit", en: Revista Anales del Seminario de Historia de la Filosofia, v. XXXVII, 1 (2020), pp. 99-111. https://doi.org/ 10.5209/ ashf.62898

Calle Zapata, M., "Martin Heidegger y el intento por pensar la esencia de la técnica como una reorientación” en: el Ethos, Revista Perseitas, v. IV, 1 (2016), pp. 41-61. https://doi.org/ 10.21501/23461780.1803

Catoggio, L., y D. Parente, "Angustia y aburrimiento. Reflexiones sobre el desplazamiento temático en el primer Heidegger”, en: Ergo, 22-23 (2008), pp. 7-24. 
De Lara, F., "El gesto político de las filosofias de Heidegger. Contribución a un debate actual", en: Veritas, 31 (2014), pp. 73-86. https://doi.org/10.4067/ S0718-92732014000200004

Di Pego, A., "En torno al pensamiento: la disputa de Hannah Arendt con Martin Heidegger, Tópicos”, en: Revista de Filosofia, 56 (2019), pp. 197-235. https:// doi.org/10.21555/top.v0i56.968

Von Falkenhayn, K., Augenblick und Kairos. Zeitlichkeit im Frühwerk Martin Heideggers, Berlin: Duncker \& Humblot, 2003.

Ferrer Santos, U., "De la autodeterminación del existente a la aceptación del ser en la Analítica existencial heideggeriana”, en: Investigaciones fenomenológicas, 3 (2001), pp. 179-193. https://doi.org/10.5944/rif.3.2001.5425

Garrido, Á., "Instante y situación. Problematización de la relación entre instante y situación en torno a Ser y tiempo de Martin Heidegger", en: Ágora. Papeles de filosofía, v. XXXVII, 2 (2018), pp. 53-75.

Heidegger, M., Sein und Zeit, Fráncfort: Klostermann,1927a.

Heidegger, M., Ser y tiempo, Gaos, J. (trad.), Buenos Aires: Fondo de Cultura Económica, 2000.

Heidegger, M., Die Grundprobleme der Phänomenologie, Fráncfort: Klostermann, 1927b .

Heidegger, M., Los problemas fundamentales de la fenomenología, Madrid: Trotta, 2000.

Heidegger, M., Was ist Metaphysik?, en: Von Herrmann, F.-W. (ed.), Wegmarken (19191961), Fráncfort: Klostermann, 2004.

Heidegger, M., ¿Qué es metafísica?, Cortés, H. y A. Leyte (trads.), Madrid: Alianza Editorial, 2007.

Heidegger, M., Die Grundbegriffe der Metaphysik. Welt - Endlichkeit - Einsamkeit (Winter semester 1929/30), Von Herrmann, F.-W. (ed.), Fráncfort: Klostermann, 2004.

Heidegger, M., Los conceptos fundamentales de la metafisica. Mundo, finitud, soledad, Ciria Cosculluela, J.A. (trad.), Madrid: Alianza Editorial, 2007.

Von Herrmann, F.-W., La segunda mitad de Ser y Tiempo. Sobre los problemas fundamentales de la Fenomenología de Heidegger, Madrid: Trotta, 1997.

Kawahara, E., "Heideggers Auslegung der Langweile”, en: Wisser, R. (ed.), Unterwegs im Denken., Friburgo y Múnich: Karl Alber Verlag, 1987, pp. 87-110.

Lythgoe, E., "Modos de ser y temporalidad en la analitica existenciaria", en: Revista de Filosofia Aurora, v. XXVII, 2 (2002), pp. 259-285.

Lythgoe, E., "Disposición afectiva y temporalidad en Martin Heidegger entre 1927 y 1930", en: Revista. de Filosofia Aurora, v. XXVI, 39 (2014), pp. 759-775. https:// doi.org/10.7213/aurora.26.039.AO03

Pocai, R., Heideggers Theorie der Befindlichkeit, Friburgo/Múnich: Karl Alber Verlag, 1996.

Redondo Sánchez, P., Filosofar desde el temple de ánimo: la 'experiencia fundamental' y la teoria del 'encontrarse' en: Heidegger, Salamanca: Ediciones Universidad de Salamanca, 2005.

Richardson, W., Heidegger: Through Phenomenology to Thought, La Haya: Martinus Nijhoff, 1967. 
Santiesteban, L., "Heidegger: la relación de su pensar con la antropología", en: Signos Filosóficos, v. VII, 13 (2005), pp. 73-103.

Santiesteban, L., Heidegger y la ética, Ciudad de México: Aldus, 2009.

Sartre, J.P., El ser y la nada, Buenos Aires: Losada, 2017.

Van Dijk, R., "Grundbegriffe der Metaphysik. Zur formalanzeigenden Struktur der philosophischen Begriffen bei Heidegger”, en: Heidegger Studien, 7 (1991), pp. 89-110. https://doi.org/10.5840/heideggerstud199176

Vélez López, G., "Peso de la existencia y dificultad hermenéutica en la génesis del pensamiento de Heidegger", en: Co-herencia, v. VI, 10 (2009), pp.113-124.

Vélez López, G., "La semilla del humanismo Moralidad y ontología fundamental en Heidegger" en: Co-herencia, v. X, 20 (2014), pp. 121-139. https://doi. org/10.17230/co-herencia.11.20.6

Ward, K., Augenblick. The Concept of the 'Decisive Moment' in 19th - and 20th Century Western Philosophy, Aldershot/Burlington: Ashgate Publishing, 2008. 